was reported in 5 (28\%). There were no changes along the ten years reviewed.

Conclusion Most deaths in infants with HIE are preceded by a clear decision of W/LT, usually within the first three days of life. The W/LT may last usually a few hours to days. We did not find changes surrounding end of life during the decade.

\section{END-OF-LIFE ETHICAL ISSUES: PAEDIATRIC INTENSIVISTS DIFFER FROM NON-INTENSIVIST PAEDIATRICIANS}

doi:10.1136/archdischild-2012-302724.0179

'S Grosek, ${ }^{2} \mathrm{M}$ Orazem, ${ }^{2} \mathrm{M}$ Kanic, ${ }^{3} \mathrm{G}$ Vidmar, ${ }^{4} \mathrm{U}$ Groselj. ${ }^{1}$ Department of Pediatric Surgery and Intensive Care, University Medical Centre Ljubljana; ${ }^{2}$ Medical Faculty, University of Ljubljana; ${ }^{3}$ University Rehabilitation Institute; ${ }^{4}$ University Children Hospital, Ljubljana, Slovenia

Background Ethical issues often arise near the end-of-life (EOL) because of concerns about what is appropriate care and who should decide. Differences may exist between paediatric intensivists and non-intensivist paediatricians.

Aim The aim of study was to assess if the approach toward EOL ethical issues differ between paediatric intensivists and non-intensivist paediatricians.

Methods Questionnaire was given to intensivists working in the Slovene paediatric ICUs and to paediatricians participating at a yearly meeting on issues in critically ill child. The questionnaire was assessing the opinion about EOL ethical issues and experiences with them.

Results Twenty-four out of 30 Slovene paediatric and neonatal intensivists and 35 out of 65 non-intensivist paediatricians responded. The average ages in both groups were 42 years. Over $90 \%$ of intensivists as compared to less than a third of non-intensivists knew whom to counsel in ethical dilemmas ( $p=0.004)$. Eightythree percent of intensivists accepted withdrawing of treatment as ethically appropriate as compared to $53 \%$ of the non-intensivists $(p=0.0002)$. Do-not-resuscitate order was always followed by $59 \%$ of paediatric intensivists. Neither group found physician's religious and cultural beliefs to be very important in decision-making process (69\% and 66\%).

Conclusions Substantial differences existed between paediatric intensivists and non-intensivis paediatrician in EOL ethical issues. Since only a third of non-intensivist paediatricians knew whom to counsel when facing an ethical dilemma and only around half of them accepting withdrawing of care as ethically appropriate, better ethical training is needed. Interestingly, neither group considered physician's religious and cultural beliefs to be very important.

\section{PARENT'S PERCEPTION OF END OF LIFE IN BRAZILIAN PEDIATRIC INTENSIVE CARE UNITS}

doi:10.1136/archdischild-2012-302724.0180

1,2P Lago. 'Pediatric Intensive Care Unit, Hospital de Clinicas de Porto Alegre, ${ }^{2}$ Pediatrics, UFCSPA, Porto Alegre, Brazil

Objective Assess the parents perception of patients who died in the Pediatric Intensive Care Unit (PICU) about the care given by health staff in the moments preceding the children's death.

Methods Exploratory-descriptive study with a qualitative approach. Settings: 2 PICU in southern Brazil. Subjects: 15 parents of children who died from April to September 2008. Data collection was performed through 3 steps:

a. The researchers contacted the parents by phone call to invite them to attend to the hospitals,

b. The doctors who assisted the children clarified doubts about the therapy offered.

c. A semi-structured interview, was carried out by researchers who had not participated of the care.
Results The analysis resulted in 4 categories:

a. the moment of death in the PICU;

b. talking with the attending physicians;

c. parental involvement in decision making;

d. parental participation in research

The results show that parents lack a peaceful environment where they can adequately carry out the goodbyes at the time of death of their children. They emphasized the solidarity provided by the nursing staff at this point and the little involvement of the medical team. The opportunity to revisit the process of their children's death with the team physician was considered positive. Parents felt that they did not have an effective participation in decision taking. Conclusion The research shows that the difficulty of communication between health staff and parents is a factor that impacts negatively on the decision taking and grieving processes.

\section{RESUSCITATION OF NEONATES AT 23 WEEKS GESTATIONAL AGE: A COST-EFFECTIVENESS ANALYSIS IN THE UNITED STATES}

doi:10.1136/archdischild-2012-302724.0181

${ }^{1} \mathrm{KR}$ Robertson, ${ }^{2} \mathrm{JC}$ Partridge, ${ }^{2} \mathrm{E}$ Rogers, ${ }^{1} \mathrm{G}$ Ottaviani, ${ }^{3} \mathrm{AG}$ Cahill, ${ }^{4} \mathrm{AB}$ Caughey. ${ }^{1}$ School of Medicine; 'Pediatrics, University of California, San Francisco, San Francisco, CA; ${ }^{3}$ Obstetrics and Gynecology, Washington University School of Medicine, St. Louis, MO, ${ }^{4}$ Obstetrics and Gynecology, Oregon Health Sciences University, Portland, OR, USA

Background and aims The appropriateness of intensive care for extreme prematurity continues controversial. In neonatal intensive care, an increasingly common choice is whether or not to resuscitate at 23 weeks gestational age. We sought to investigate whether such an intervention is cost effective.

Methods A decision analytic model was designed comparing resuscitation vs. non-resuscitation from a societal perspective for pre-term deliveries at 23 weeks. Estimates of death (74\%) and neurodevelopmental disability (84-91\%) in the setting of resuscitation were taken from the existing literature. Utilities were applied to discounted life expectancy to generate OALYs. All costs and OALYs were discounted at $3 \%$. A cost-effectiveness threshold of $\$ 100,000$ per OALY was utilized. Sensitivity analysis included univariate and bivariate comparisons and Monte Carlo simulations.

Results Non-resuscitation is the dominant strategy, as it is both less expensive ( $\$ 71,036$ v. $\$ 259,358$ ) and more effective (24.7 OALYs v. 24.4 OALYs). While resuscitation would lead to 240 live infants, in a theoretical cohort of 1,000 cases, there would be 100 severely disabled, 70 moderately disabled, and 90 non-disabled survivors. In univariate sensitivity analysis, non-resuscitation was the costeffective strategy at all reasonable ranges of the inputs for the cost of NICU care and risk of mortality and disability.

Conclusions From a societal perspective, it does not appear costeffective to resuscitate 23 week neonates over a wide range of assumptions. In our model, even if NICU care is free, resuscitation is not cost effective at baseline due to extreme long-term costs.

\section{NEC PATHOGENESIS - NEWS FROM PRETERM PIGS}

doi:10.1136/archdischild-2012-302724.0182

PT Sangild. Univ Copenhagen, Frederiksberg, Denmark

Nutritional, microbiological and immunological dysfunctions all play a role in NEC etiology but the relationship among these determinants is not understood. The preterm gut is very sensitive to enteral feeding which may either promote gut adaptation or induce gut dysfunction via bacterial overgrowth and inflammatory reactions. Tumor necrosis factor alpha, toll-like receptors and heatshock proteins are identified among the immunological components of the early mucosal dysfunction. It remains difficult, however, to 
distinguish the early initiators of NEC from the later consequences of the disease pathology. To elucidate the mechanisms and identify clinical interventions, animal models showing spontaneous NEC development may help. In this review, we summarize some recent results from studies on preterm pigs during the early feedinginduced mucosal dysfunction and later NEC development. We show that introduction of suboptimal enteral formula diets, coupled with parenteral nutrition, predispose to disease, while advancing amounts of mother's milk from birth protects against disease. Hence, the transition from parenteral to enteral nutrition shortly after birth plays a pivotal role to secure gut growth, digestive maturation and an appropriate response to bacterial colonization in the sensitive gut of preterm neonates. Ongoing studies in preterm pigs aim to identify the optimal time, amount and diet of the first enteral milk that best secure both early gut adaptation and later body growth and health.

\section{PREVENTION OF NECROTISING ENTEROCOLITIS}

doi:10.1136/archdischild-2012-302724.0183

1.2S Patole. 'Neonatal Paediatrics, KEM Hospital for Women; ${ }^{2}$ Centre for Neonatal Research and Education, University of Western Australia, Perth, WA, Australia

Necrotising enterocolitis (NEC) is a potentially disastrous illness that occurs in $6-8 \%$ of preterm (gestation $<32$ weeks) very low birth weight neonates. The mortality $(\sim 25 \%)$ and morbidity of $\geq$ Stage II NEC (e.g. need for surgery, survival with short bowel syndrome with protracted feed intolerance, complications of prolonged dependence on parenteral nutrition, recurrent infections, and prolonged hospital stay) is significant. The incidence (10-12\%), mortality (40-45\%), and morbidity including need for surgery, and risk of long-term neurodevelopmental impairment after surviving surgery for NEC is worse in extremely low birth weight neonates. The economic burden of NEC is substantial ( $~ 500$ million to 1 billion dollars per year in the USA) considering the prolonged hospital stay due to the associated complications. NEC has become one of the common causes of death in preterm neonates surviving the first week of life. The pathogenesis of NEC remains poorly understood despite decades of research. Prevention of prematurity, the single most important risk factor for the illness, has proved to be a difficult task. The absolute number of preterm neonates at risk for the illness has increased with advances in neonatal intensive care. Prevention of NEC has thus become a priority. The well established (e.g. antenatal glucocorticoids, early preferential feeding with breast milk, standardised feeding protocols) as well as newer strategies (e.g. probiotics, prebiotics) for primary as well as potentially secondary (e.g. pentoxifylline, bosentan) prevention of NEC will be reviewed.

\section{INTRAPERITONEAL CYTIDINE 5'-DIPHOSPHOCHOLINE (CDP-CHOLINE) ADMINISTRATION REDUCES THE SEVERITY OF INTESTINAL INJURY IN A NEONATAL RAT MODEL OF NECROTIZING ENTEROCOLITIS}

doi:10.1136/archdischild-2012-302724.0184

'M Cetinkaya, ${ }^{2} \mathrm{M}$ Cansev, ' $1 \mathrm{~F}$ Cekmez, ' $\mathrm{C}$ Tayman, ' $\mathrm{FE}$ Canpolat, ${ }^{2} \mathrm{M}$ Kafa, ${ }^{3} \mathrm{~S}$ Uysal, ${ }^{1} \mathrm{SU}$ Sarıı. ' GATA Teaching Hospital, Ankara; ${ }^{2}$ Uludağ University Medical Faculty, Bursa; ${ }^{3}$ Fatih University Medical Faculty, Ankara, Turkey

Background The aim of this study was to evaluate the possible cytoprotective effect of CDP-choline treatment on intestinal cell death and apoptosis in a neonatal rat model of NEC.

Methods A total of 30 newborn pups were divided equally into 3 groups as follows: Control, NEC, and NEC+CDP-choline groups. NEC was induced by enteral formula feeding, exposure to hypoxiahyperoxia and cold stress. CDP-choline was administered intraperitoneally at a dose of $300 \mathrm{mg} / \mathrm{kg} /$ day for 3 days starting from the first day of life. Macroscopical, histopathological, ibflammatory markers, caspase- 3 expression and apoptosis were evaluated on the gut samples. Activities of xanthine oxidase, superoxide dismutase, glutathione peroxidase, malondialdehyde and myeloperoxidase were determined.

Results Median clinical sickness score, macroscopic gut assessment score and intestinal injury score were significantly improved in pups in NEC+CDP-choline group. In contrast, median apoptosis score was significantly higher in NEC group compared with NEC+CDP-choline group. Proinflammatory cytokine concentrations (IL-1 $\beta$, IL- 6 and TNF- $\alpha$ ) and caspase-3 expression in the intestinal tissue of the NEC+CDP-choline group were significantly lower. Moreover, tissue GSH-Px and SOD activities were preserved, whereas tissue MDA content, $\mathrm{MPO}$ and $\mathrm{XO}$ activities were significantly lower in NEC+CDP-choline group.

Conclusion This is the first study to report beneficial effects of CDP-choline treatment on intestinal injury in a neonatal rat model of NEC. Intraperitoneal CDP-choline administration significantly reduced clinical sickness score, ameliorated macroscopic and histopathological intestinal injury, reduced the inflammation and decreased apoptosis. These data suggest that, CDP-choline may be used as an effective therapeutic agent for prevention of NEC.

\section{MESENTERIC ARTERY REACTIVITY AND SMALL INTESTINE MORPHOLOGY IN A CHICKEN MODEL OF HYPOXIA- INDUCED FETAL GROWTH RESTRICTION}

doi:10.1136/archdischild-2012-302724.0185

${ }^{1}$ RM Moonen, ${ }^{2}$ CG Kessels, ${ }^{2}$ E Villamor. 'Pediatrics, Atrium Medical Center Parkstad, Heerlen; ${ }^{2}$ Pediatrics, Maastricht University Medical Center, Maastricht, The Netherlands

Background and aims Infants with intrauterine growth retardation are prone to intestinal dysfunction which is manifested by feeding intolerance and, in the most severe cases, necrotizing enterocolitis. The morphological and molecular mechanisms that lead to these complications are not completely understood and suitable experimental models are necessary. We aimed to characterize mesenteric artery $(\mathrm{MA})$ reactivity, small intestine morphometry and intestinal expression of VEGF in a chicken model of hypoxiainduced fetal growth restriction.

Methods Chicken embryos (15 and 19 days) and hatchlings $\left(<3\right.$-h-old and 1-d-old) were incubated under hypoxic $\left(15 \% \mathrm{O}_{2}\right.$ from day 0 to day 19 of incubation) or normoxic conditions. Vascular reactivity was studied using wire miography. Intestinal morphometry was assessed in hematoxyline-eosine-stained sections. The expression of VEGF mRNA was determined by RTPCR analysis.

Results Hypoxia altered the responsiveness of chicken embryo MAs to acetylcholine, the NO donor sodium nitroprusside and the constrictor polypeptide ET-1. However, the majority of these alterations, with the exception of the hyperresponsiveness to ET-1, were not present in the hypoxic hatchlings. When intestinal histology was analyzed, subtle hypoxia-induced changes were noted in the muscularis propia and the villi from the hatchlings. Hypoxic incubation also diminished the expression of VEGF mRNA in the terminal ileum of the hatchlings.

Conclusions Chronic moderate hypoxia during incubation results in subtle but significant alterations in chicken MA reactivity, small intestine morphology and VEGF expression. Whether these alterations may have a direct effect on the functional status of the intestine remains to be investigated.

\section{PROTECTIVE EFFECTS OF COLCHICINE IN AN EXPERIMENTAL MODEL OF NECROTIZING ENTEROCOLITIS IN NEONATAL RAT}

doi:10.1136/archdischild-2012-302724.0186 